\title{
miR-129-3p controls centrosome number in metastatic prostate cancer cells by repressing CP110
}

\author{
Irene V. Bijnsdorp ${ }^{1}$, Jasmina Hodzic ${ }^{2}$, Tonny Lagerweij ${ }^{3}$, Bart Westerman ${ }^{3}$, Oscar \\ Krijgsman $^{4,5}$, Jurjen Broeke ${ }^{6}$, Frederik Verweij ${ }^{4}$, R. Jonas A. Nilsson ${ }^{3,7}$, Lawrence \\ Rozendaal ${ }^{4}$, Victor W. van Beusechem ${ }^{2}$, Jeroen A. van Moorselaar ${ }^{1}$, Thomas $^{2}$ \\ Wurdinger ${ }^{3,8, *}$, Albert A. Geldof ${ }^{1, *}$ \\ ${ }^{1}$ Department of Urology, VU University Medical Center, Amsterdam, The Netherlands \\ ${ }^{2}$ Department of Medical Oncology, VU University Medical Center, Amsterdam, The Netherlands \\ ${ }^{3}$ Department of Neurosurgery, VU University Medical Center, Amsterdam, The Netherlands \\ ${ }^{4}$ Department of Pathology, VU University Medical Center, Amsterdam, The Netherlands \\ ${ }^{5}$ Department of Molecular Oncology, The Netherlands Cancer Institute, Amsterdam, The Netherlands \\ ${ }^{6}$ Center for Neurogenomics and Cognitive Research, VU University, Amsterdam, The Netherlands \\ ${ }^{7}$ Department of Radiation Sciences, Oncology, Umeå University, Umeå, Sweden \\ ${ }^{8}$ Department of Neurology, Massachusetts General Hospital, Harvard Medical School, Boston, MA, USA \\ *These authors have contributed equally to this work \\ Correspondence to: Irene V. Bijnsdorp, e-mail: iv.bijnsdorp@vumc.nl \\ Keywords: prostate cancer, centrosome, CP110, miR-129-3p, metastasis \\ Received: October 10,2015 Accepted: February 02, $2016 \quad$ Published: February 23, 2016
}

\section{ABSTRACT}

The centrosome plays a key role in cancer invasion and metastasis. However, it is unclear how abnormal centrosome numbers are regulated when prostate cancer (PCa) cells become metastatic. CP110 was previously described for its contribution of centrosome amplification (CA) and early development of aggressive cell behaviour. However its regulation in metastatic cells remains unclear. Here we identified miR129-3p as a novel metastatic microRNA. CP110 was identified as its target protein. In PCa cells that have metastatic capacity, CP110 expression was repressed by miR129-3p. High miR-129-3p expression levels increased cell invasion, while increasing CP110 levels decreased cell invasion. Overexpression of CP110 in metastatic PCa cells resulted in a decrease in the number of metastasis. In tissues of PCa patients, low CP110 and high miR-129-3p expression levels correlated with metastasis, but not with the expression of genes related to EMT. Furthermore, overexpression of CP110 in metastatic PCa cells resulted in excessive-CA (E-CA), and a change in F-actin distribution which is in agreement with their reduced metastatic capacity. Our data demonstrate that miR-129-3p functions as a CA gatekeeper in metastatic PCa cells by maintaining pro-metastatic centrosome amplification (CA) and preventing antimetastatic E-CA.

\section{INTRODUCTION}

Prostate cancer $(\mathrm{PCa})$ is the second most common cause of cancer related death in males in the Western countries [1]. For patients with metastatic PCa no curative therapy is available. The progression of localized PCa to metastatic variants involves multiple sequential steps, including cell migration and invasive growth [2].
The centrosome is an organelle that serves as the main microtubule organizing centre of the cell [3]. Prostate cancer cells frequently show centrosome amplification (CA; 3-5 centrosomes/cell). CA leads to promotion of tumor growth through induction of chromosomal missegregation and modulation of the microtubule cytoskeleton, causing enhanced directional migration and invasion of malignant cells [4-6]. CA 
conveys cytoskeletal advantages that enhance cell polarization, Golgi-dependent vesicular trafficking, and stromal invasion [4-6]. CP110 is an evolutionary conserved centrosomal protein important for centrosome functioning [7-9], controlling cell migration [10]. CP110 overexpression caused CA and increased the invasive phenotype of cells [7, 11]. However, the actual contribution of CP110 in PCa metastasis has not been described.

MicroRNAs (miRNAs) are small non-coding RNAs that suppress translation of target mRNAs, and tightly regulate numerous cellular processes [12]. In cancer cells miRNAs are often deregulated [13], including in PCa cells [14, 15]. Multiple studies identified miRNAs correlating to $\mathrm{PCa}$ metastasis or PCa survival [16-18]. In non-neoplastic cells, CP110 expression can be regulated by miR-34/449 [19] and miR-129-3p [10]. To determine the potential contribution of miRNAs in PCa cells to the regulation of CP110 and metastasis, we performed miRNA profiling of metastatic and non-metastatic PCa tissues. We confirmed the correlative expression of miRNAs previously associated with metastatic $\mathrm{PCa}$, including miR-34 [17]. Moreover we observed high expressiojn of miR-129-3p in metastatic PCa cells. MiR-129$3 \mathrm{p}$ has not been previously associated with $\mathrm{PCa}$ metastasis. MiR-129-3p decreased the expression of the centrosomal protein CP110. Lack of CP110 inhibition led to excessive centrosome amplification (E-CA; > 5 centrosomes/cell), increased E-cadherin expression, deregulation of the F-actin cytoskeleton and diminished invasion and metastasis. We show that centrosome regulation in metastatic $\mathrm{PCa}$ cells differs from that in early stages of prostate cancer development. When cells acquire a metastatic capacity, CP110 levels are reduced to control centrosome number and thereby prevent E-CA.

\section{RESULTS}

\section{miR-129-3p is overexpressed in metastatic PCa}

To identify miRNAs involved in PCa metastasis, we used the Dunning rat PCa progression model [20]. Two successive stages of progressive PCa were selected, i.e. AT-1 (AT1, locally invasive PCa), and MatLyLu (MLL, metastatic PCa) (Figure 1a). RNA was isolated from AT1 and MLL PCa tissues and miRNA expression array analyses were performed to determine which miRNAs are differentially expressed (Supplementary Table S1). The obtained miRNA expression profiles were subjected to (unsupervised) hierarchical cluster analyses (Figure 1b-1c). Only one miRNA (miR665; Supplementary Table S1) was downregulated in metastatic MLL as compared to locally invasive AT1 PCa tissues $(>2$-fold, $\mathrm{p}<0.05)$. In contrast, in MLL tissues we identified in total 24 upregulated miRNAs ( $>2$-fold, $\mathrm{p}<0.05$; Supplementary Table S1). The pro-metastatic miRNAs that were increased most include the previously identified miR-34c [17], and a newly identified miRNA, miR-129-3p (Figure 1c; Supplementary Table S1). RTPCR was used to validate the differential expression levels of pro-metastatic miR-129-3p and miR-34c (3and 9-fold upregulation in MLL compared to AT-1 tumor tissues, respectively), (Figure 1d). Of note, the miR129-3p and miR-34c levels in rat MLL lung metastases (MLL-LM) were similar to the levels in rat MLL primary PCa tissue (MLL-P). In addition, we performed RNAseq on non-neoplastic human prostate epithelial cells (prEC) and prostate fibroblasts (prSC) and the human PCa cell cultures LNCaP and PC3 (Figure 1e). LNCaP and PC3 are both cell lines derived from metastasis, however when grown as subcutaneous xenografts they do not demonstrate metastatic capacity [21]. Although PC3 in other systems, such as after intracardiac injection or injection into bone can be used to study metastasis [22], the miRNA expression of PC3 is consistent with the non-metastatic potential of PC3 when grown in subcutaneous models, i.e. low miR-34c and miR129-3p expression. Expression of these miRNAs was confirmed by RT-PCR in cultured human LNCaP, PC3 and rat MLL PCa cells (Figure 1f). Previously, miR-129$3 p$ was shown to functionally repress the translation of the centrosomal protein CP110. Because this miRNA has not been described before in metastatic $\mathrm{PCa}$, it was highly differential, and is potentially related to CP110, we selected this miRNA for further evaluation. Figure $1 \mathrm{~g}$ shows the two complementary binding sites of miR-129$3 p$ in the $3^{\prime}$-UTR of the human and rat CP110 mRNAs. To validate that miR-129-3p represses CP110 in $\mathrm{PCa}$ cells, we overexpressed miR-129-3p in human PC3 and rat MLL cells by transfection of a miR-129-3p mimic. We indeed measured a reduction in CP110 protein levels by Western blot. Conversely, inhibition of miR-129$3 p$ by transfection of anti-sense molecules resulted in increased CP110 protein levels (Figure 1h, left panel). In addition, we transfected PC3 and MLL cells with a CP110 $33^{\prime}$ UTR expression cassette without miR-129-3p $3^{\prime}$-UTR. Because the miR-129-3p binding site is located on the 3'-UTR this construct enables CP110 expression independently of miR-129-3p (Figure 1h, right panel). In these cells, CP110 expression maintained after transfection with either miR-129-3p mimic or inhibitor, with a modest modulation which is likely caused by endogenous CP110 expression. We performed a target scan using multiple miRNA-target databases, showing a strong putative match between highly expressed miRNAs and predicted target sequences in the $3^{\prime}$ UTR of CP110. A total of $12 / 24$ miRNAs (50\%) that were upregulated in metastatic MLL tumors were predicted to have a potential binding site in CP110 (Supplementary Table S2). 


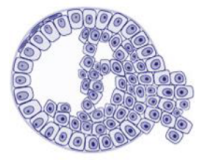

Anaplastic tumor (AT1) nonmetastatic capacity

C

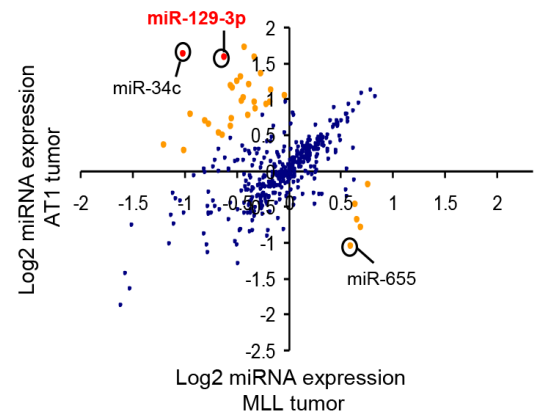

d
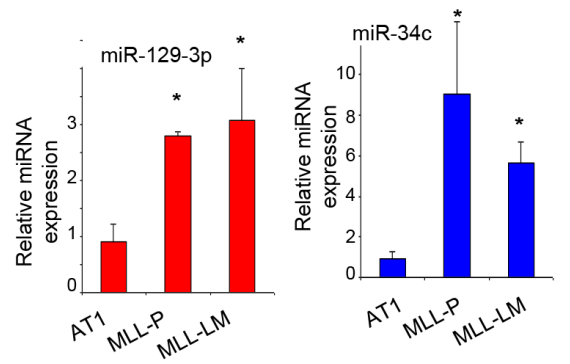

e

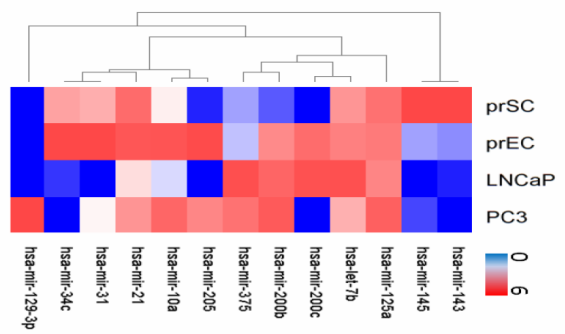

g

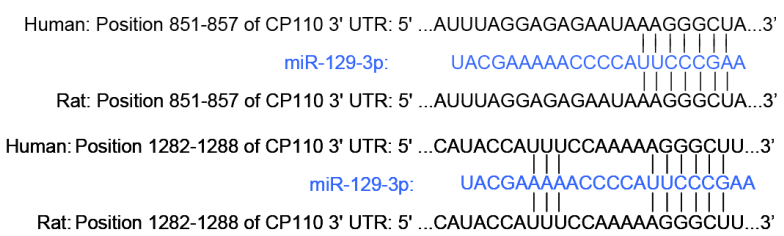

b

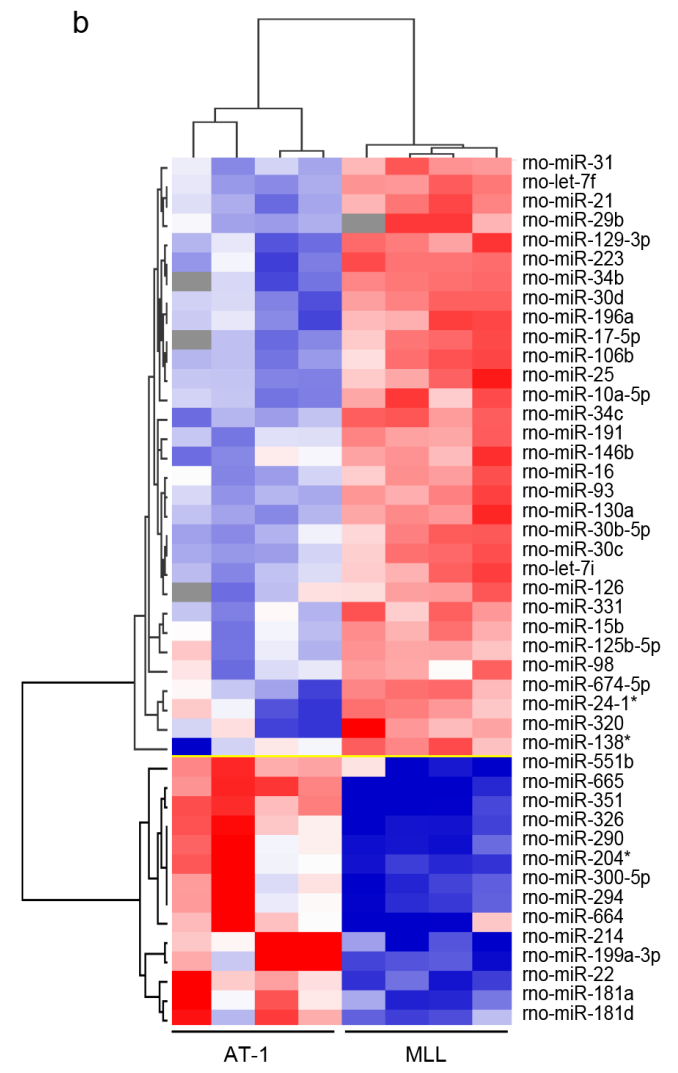

f

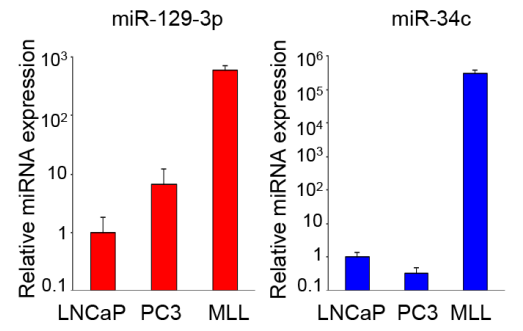

$\mathrm{h}$

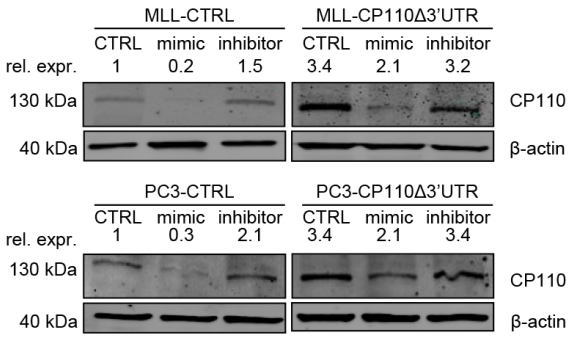

Figure 1: miR-129-3p is overexpressed in metastatic PCa. a. Characteristics of the selected variants of the PCa Dunning progression model. b. Hierarchical cluster analysis of miRNA expression (miRNA microarray) of AT1 and MLL tumor tissues. c. Comparative expression analysis (microarray) between AT1 and AT1 and MLL tumor tissues. Each dot indicates one miRNA showing the average log2 expression value from four independent tissue samples. Red indicates $>4$ fold change, orange indicates $2-4$ fold change, $p<0.05$. d. RT-PCR validation of miRNA expression levels of miR-129-3p and miR-34c in the tumor tissues of AT1 and MLL. Values represent means of three independent tumor tissue samples \pm s.e.m. MLL-P = MLL primary tissue; MLL-LM = MLL lung metastasis tissue. e. RNAseq analysis of selected miRNAs in in vitro cultures of non-cancerous prSC, prEC and the PCa cell lines PC3 and LNCaP. Clustering of normalized, log transformed RNAseq expression data was performed using Cluster 3.0 software and visualized using Java treeview f. Relative miRNA expression levels of miR-129-3p and miR-34c in LNCaP, PC3 and MLL cells. Values represent means of three independent experiments \pm s.e.m. g. Confirmed CP110 3'-UTR binding sites for miR-129-3p. h. CP110 protein expression in cells transfected with a CP110 $\Delta^{\prime} 3 \mathrm{UTR}$ rescue construct, with or without transfection with miR-129-3p mimics or inhibitors. ${ }^{*} \mathrm{p}<0.05$, T-test. 


\section{CP110 is repressed by miR-129-3p and downregulated in metastatic PCa}

The clinical relevance of miR-129-3p, and its target protein $\mathrm{CP} 110$ in $\mathrm{PCa}$ was determined by measuring their expression levels in human $\mathrm{PCa}$ tissues. CP110 protein expression levels were determined in tissues of locally confined $\mathrm{PCa}(\mathrm{n}=24)$ and $\mathrm{PCa}$ tissues from patients with metastatic disease $(\mathrm{n}=17)$ obtained by radical prostatectomy (Figure 2a-2b), showing significant downregulation of CP110 in metastatic PCa tissues $(\mathrm{p}<0.0002)$ compared to local PCa tissues. Furthermore, miR-129-3p expression levels were significantly increased in metastatic $\mathrm{PCa}$ as compared to localized PCa tissues $(\mathrm{p}<0.0002)$ (Figure $2 c)$. The increase in miR-129-3p $(\mathrm{p}<0.05)$ and decrease in CP110 ( $<<0.01)$ expression levels in metastatic PCa were validated in independent $\mathrm{PCa}$ datasets [17, 23-26] (Figure 2d-2e). Of note, the miR-129-3p and CP110 expression levels inversely correlated with the time to biochemical $\mathrm{PCa}$ recurrence after surgery (Figure 2f-2g).

\section{miR-129-3p and CP110 regulate $\mathrm{PCa}$ metastasis}

The functional role of miR-129-3p and CP110 in $\mathrm{PCa}$ progression was examined by transfecting
miR-129-3p mimic and inhibitor into PC3, PC3CP110 $\Delta 3^{\prime}$ UTR, MLL and MLL-CP110 $\Delta 3^{\prime}$ UTR cells. The non-metastatic AT1 PCa cells do not survive in culture, therefore these cells were not included. Overexpression of CP110 $33^{\prime}$ UTR in PC3 and MLL cells resulted in a minor reduction in cell proliferation in vitro (Figure 3a), a moderate decrease in migration (Figure $3 b$ ), and a large reduction in cell invasion (Figure 3c). Overexpression of miR-129-3p in PC3 and MLL PCa cells significantly increased the invasive phenotype of these cells $(p<0.01$ and $p<0.05$, respectively), while only moderate effects on proliferation and migration were observed (Figure 3a-3c). Proliferation, migration and invasion were not affected by miR-129-3p mimics or inhibitors in cells overexpressing the CP110 $\Delta 3^{\prime}$ UTR-rescue construct. These results suggest that miR-129-3p mediates invasion of PCa cells via repression of CP110. We injected $5 \times 10^{5}$ MLL-FMCTRL or MLL-FM-CP110 $\Delta 3^{\prime}$ UTR cells with F-luciferase expression (Figure 3d) subcutaneously in Copenhagen rats $(\mathrm{n}=7$ and $\mathrm{n}=8$, respectively), and monitored tumor growth. CP110 $\Delta 3^{\prime}$ UTR overexpression did not affect the growth rate of the primary tumor in vivo (Figure 4a), but did result in less invasive growth of the primary tumor as compared to the highly invasive MLL-FM-CTRL tumors (Figure 4b-4e). MLL-FM-CTRL lymph node metastases
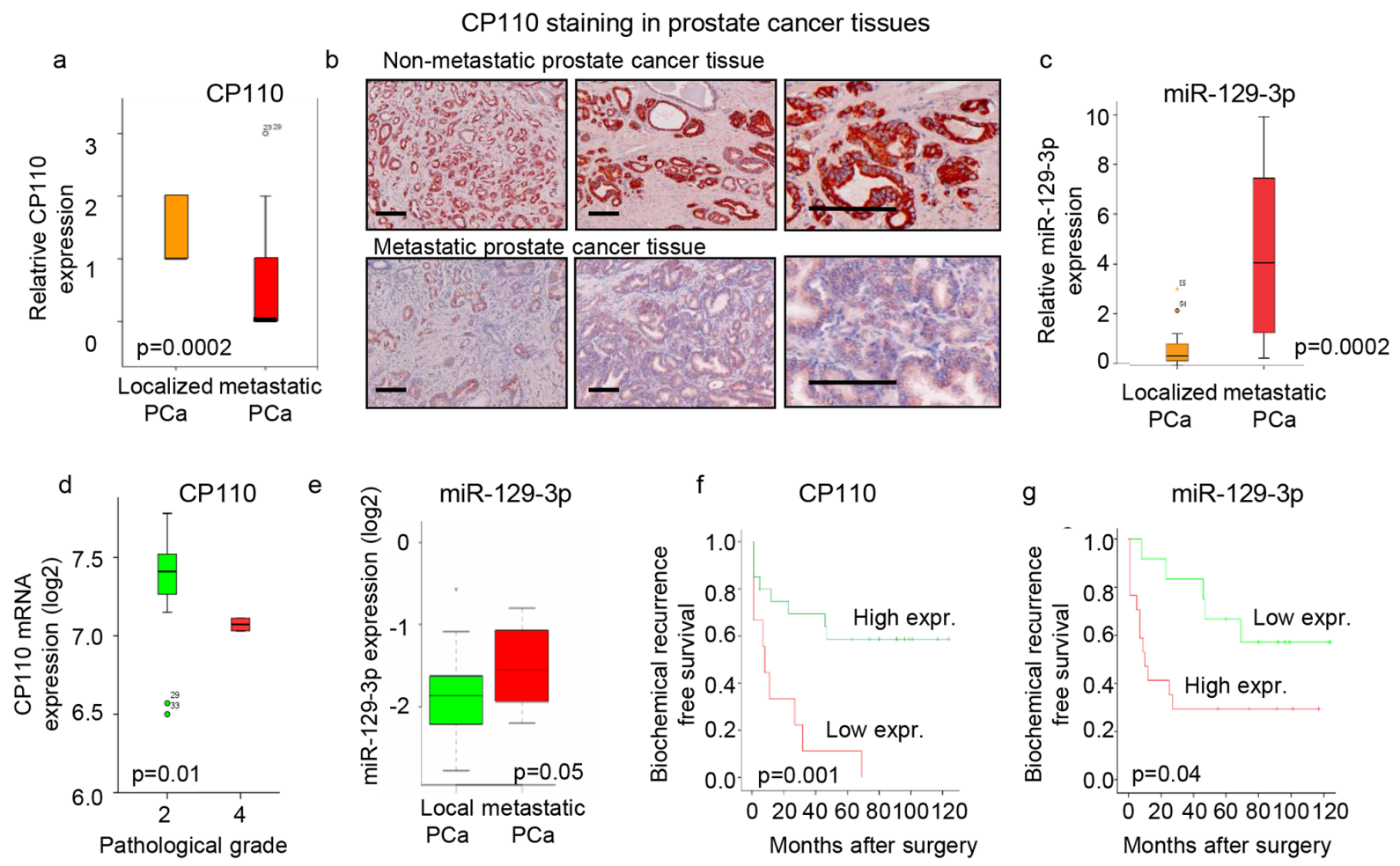

Figure 2: CP110 is repressed by miR-129-3p and downregulated in metastatic PCa. a. Quantification of CP110 protein expression in tissues from 24 patients with localized PCa and 17 metastatic PCa. Y-axis represent relative expression based on intensity score of 0-3. b. Immunostaining of CP110 protein in prostate tissues. Scale bar, $200 \mu \mathrm{m}$. c. miR-129-3p expression in tissues from patients with localized PCa and metastatic PCa. d. CP110 mRNA expression from EXPO dataset. e. miR-129-3p expression in prostate tissues from the Martens-Uzunova dataset [17]. f. Expression of CP110 (score <1) correlated to time to biochemical recurrence. g. miR-129-3p expression (expression $>4$ ) correlated to time to biochemical recurrence. 
were visible by bioluminescence imaging using a CCD camera in 5 out of 7 rats (Figure $3 \mathrm{f}-3 \mathrm{~g}$ ) and confirmed by H\&E staining (Figure $4 d$ ). This was in contrast to MLL-FM-CP110 $43^{\prime}$ UTR tumors that caused lymph node metastasis in only 1 out of 8 rats $(p<0.01)$ (Figure $4 c$ $4 d)$. These observations are indicative of a key role for CP110 in PCa invasion and metastasis. PCa metastases have been associated with down-regulation of E-cadherin, Western blot analysis of E-cadherin protein expression demonstrated that MLL-FM-CP110 $\Delta 3^{\prime} \mathrm{UTR}$ tumor tissues with low metastatic potential had elevated levels (20\%) of E-cadherin protein as compared to MLL-FM-CTRL tumor tissues with high metastatic potential (Figure 4e-4f). Moreover, the CP110 and E-cadherin expression in tissues of AT1 and MLL tumors correlated positively $\left(r^{2}=0.9117\right.$, $\mathrm{p}=0.01$; Figure $4 \mathrm{e}$ ). In addition, after transfection of MLL cells with miR-129-3p mimics we observed a decrease in E-cadherin expression in MLL cells, but not in MLLCP110 $\triangle 3^{\prime}$ UTR cells (Figure 4f). In order to determine whether EMT is involved in the observed PCa metastasis, we correlated CP110 gene expression to EMT-related genes in multiple independent clinical PCa datasets [23-26] (Figure 4g-4j and Supplementary Table S3). We selected a previously reported subset of EMT-related genes
[27]. Surprisingly, no significant correlation was measured between CP110 mRNA expression and EMT-related genes in local and metastatic PCa tissues, suggesting that the CP110-controlled PCa cells are not undergoing EMT prior to metastasis.

\section{CP110 decreases PCa invasion via centrosomes}

To determine whether CP110 affected the cytoskeleton and the centrosomes in PCa cells we analysed the F-actin cytoskeleton organization and pericentrin localization in MLL, MLL-CP110 $\Delta 3^{\prime} \mathrm{UTR}$, PC3 and PC3-CP110 43 'UTR cells. CP110 plays a key role in centrosome organization and its overexpression may cause aberrant centrosome function due to E-CA, which may decrease cell invasion and metastasis. E-CA was evaluated by staining MLL, MLL-CP110 $43^{\prime}$ UTR, PC3 and PC3-CP110 $\triangle 3^{\prime} \mathrm{UTR}$ cells for the centrosomal marker pericentrin (Figure 5a-5b). A regular cancer associated centrosome phenotype was found, with modest CA for PC3 and MLL. By contrast, the centrosomes moved towards an E-CA phenotype in MLL-CP110 $\triangle 3^{\prime} \mathrm{UTR}$ and PC3-CP110 $\Delta 3^{\prime} \mathrm{UTR}$ cells (Figure 5a-5b). The E-CA of centrosomes in MLL-CP110 43 'UTR and PC3-
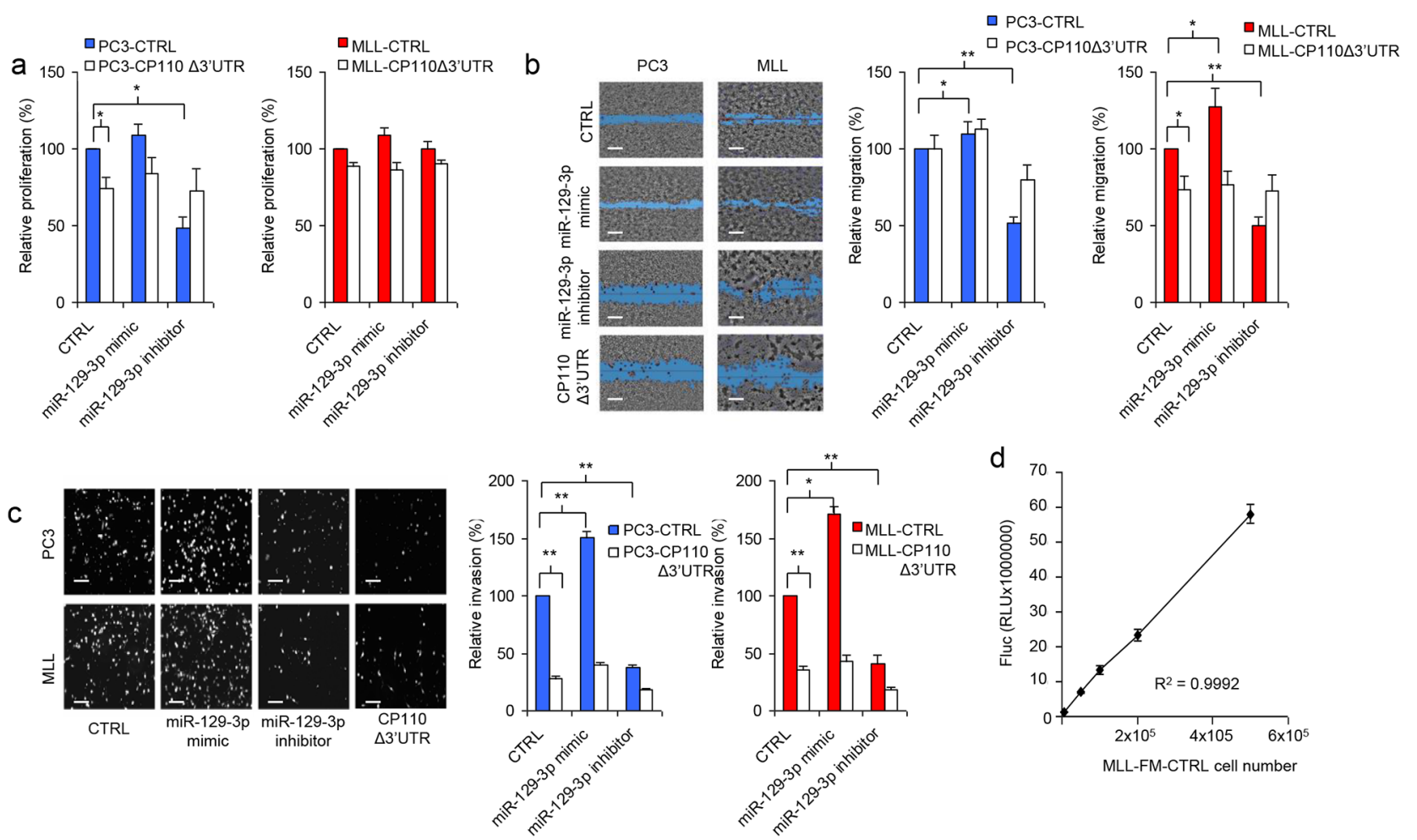

Figure 3: miR-129-3p and CP110 regulate cell invasion. a. Cell proliferation analysis after overexpression of CP110 $\Delta 3^{\prime} \mathrm{UTR}$ and/ or miR-129-3p in PC3 and MLL cells. Values represent the mean of five independent experiments \pm s.e.m. b. Representative images of cell migration analysis after overexpression of CP110 $\Delta 3^{\prime}$ UTR and/or miR-129-3p in PC3 and MLL cells, and quantification in the right panel. Values represent the mean of five independent experiments \pm s.e.m.. Scale bar, $200 \mu \mathrm{m}$. c. Representative images of cell invasion analysis after overexpression of CP110 $3^{\prime}$ UTR and/or miR-129-3p in PC3 and MLL cells, and quantification in the bottom panel.Values represent the mean of five independent experiments \pm s.e.m.. Scale bar, $200 \mu \mathrm{m}$. d. Fluc expression by MLL-FM-CTRL cells is linearly correlated to cell number. 
CP110 43 'UTR cells indicates an uncontrolled centriolar duplication rather than centrosome fragmentation due to structural instability [28]. Furthermore, centrosomes were clustered in control cells, while centrosomes were scattered in CP110 $23^{\prime}$ UTR overexpressing cells, which is also indicative for disrupted directional movement of cells [29]. To study whether the scattered overduplicated centrosomes affected cell directional movement, we stained MLL, MLL-CP110 43 'UTR, PC3, and PC3CP110 $\triangle 3^{\prime}$ UTR cells for F-actin and determined cytoskeleton structure. We observed a clear decrease in F-actin expression. Furthermore, in MLL-CP110 $\Delta 3$ 'UTR cells, the long F-actin branching structures were deregulated, with a rounded edge surface, compared to the control cells (Figure 5c-5e). This is in agreement with the observed increase in E-cadherin expression (Figure $4 \mathrm{f}$ ) and decrease in cell invasion (Figure 3c). The distinct cytoskeleton phenotypes (Figure $5 \mathrm{c}-5 \mathrm{e}$ ) are consistent with rescue of $\mathrm{CP} 110$ expression changing the F-actin distribution to a 'bold-edge' phenotype. In conclusion, our results indicate that miR-129-3p is upregulated in metastatic PCa cells resulting in repression of CP110, which is accompanied by loss of E-cadherin expression, cytoskeleton remodelling, and the formation of filopodia, endowing PCa cells with increased migration and invasion capacity (Figure 5f). a

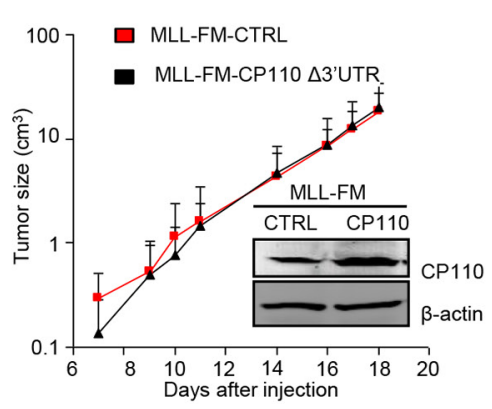

b

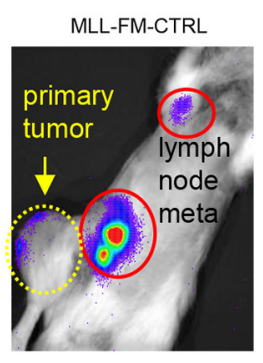

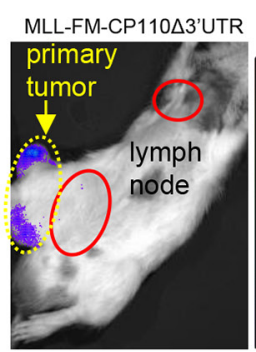

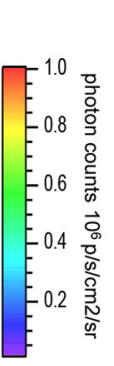

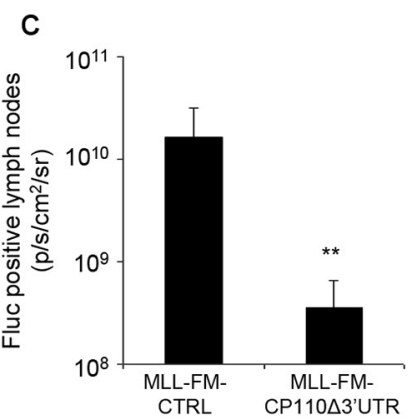

d

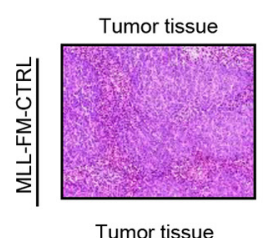

Lymph node
metastasis

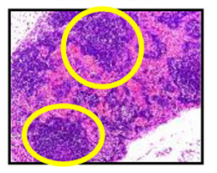

Fat tissue
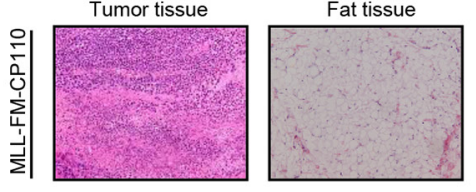

Muscle area:
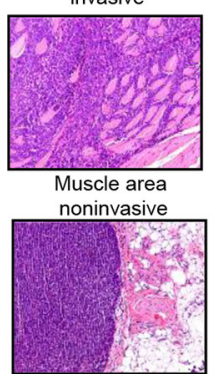

e
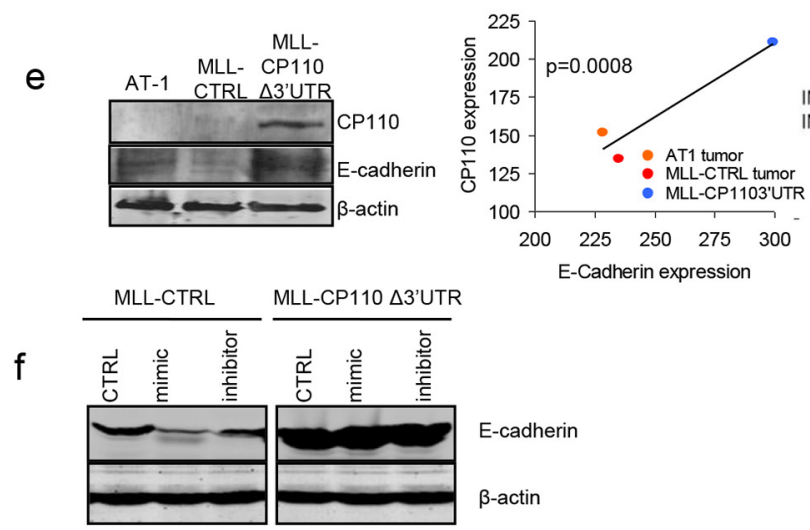

E-cadherin

$\beta$-actin
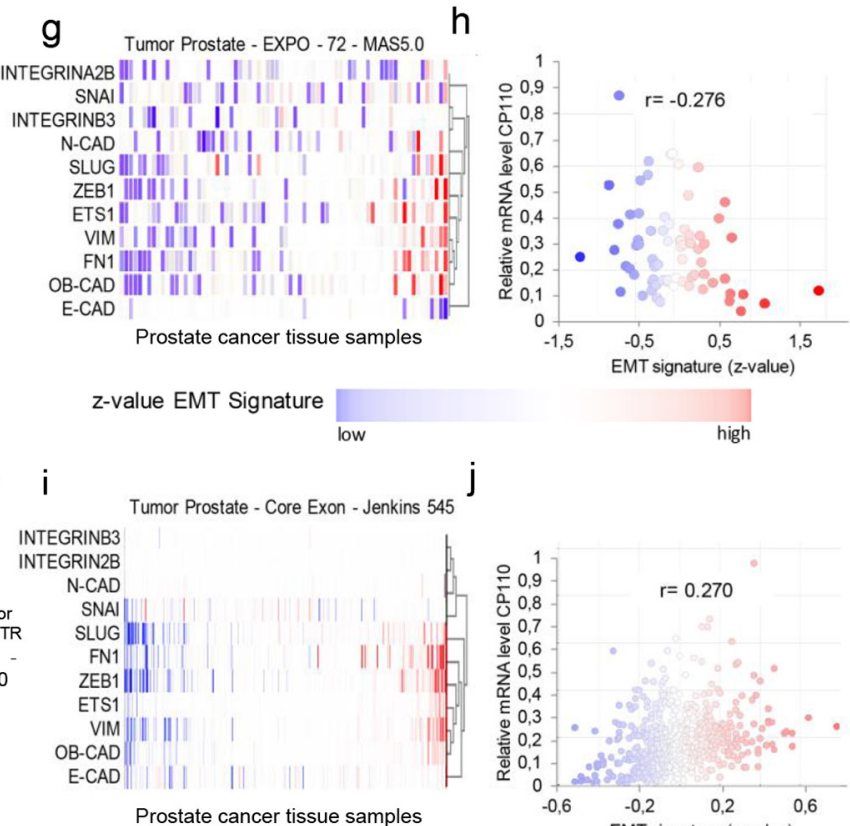

j

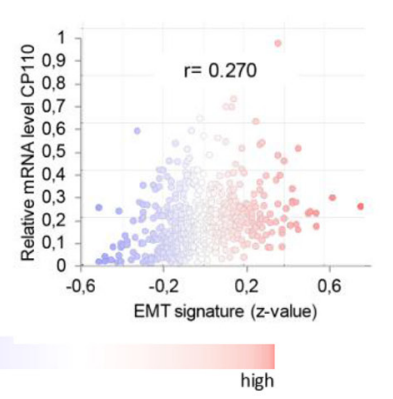

Figure 4: miR-129-3p and CP110 regulate cell metastasis. a. Primary tumor growth of MLL-FM and MLL-FM-CP110 $\Delta 3^{\prime} U T R$ cells in vivo. Insert shows CP110 Western blot of the injected cell cultures. b. Representative CCD camera images of Fluc bioluminescence imaging of lymph node metastasis, and c. Quantification of Fluc positive lymph nodes in MLL-FM-CTRL (n=7 rats) and MLL-FMCP110 $\triangle 3$ 'UTR ( $\mathrm{n}=8$ rats) cells. d. H\&E staining of tumor tissues, the invaded muscle area and positive lymph node metastasis. Only for MLL-FM cells muscle invasion and lymph node metastasis (indicated by yellow circles) were detected. e. Western blot for E-cadherin and CP110 in tissues of different progression stages of the Dunning model. f. Western blot for E-cadherin in cell cultures g-j. Correlation of CP110 with an EMT-related signature [45] as being an averaged value of all z-values of the normalized data in two clinical PCa datasets. ${ }^{*} p<0.05,{ }^{* *} p<0.01$, T-test. 


\section{DISCUSSION}

In early stages of cancer development, $\mathrm{CA}$ is a key contributor for development into more aggressive types of cancer [30]. However, in the present study we show that when aggressive prostate cancer cells acquire metastatic capacity, a feedback loop is activated that controls centrosome number. We identified miR-129-3p to be upregulated in metastatic prostate cancer cells, which negatively regulates $\mathrm{CP} 110$ expression. Rescue of CP110 expression in metastatic $\mathrm{PCa}$ cells caused E-CA and prevented invasion, and lymph node metastases. About
$50 \%$ of the miRNAs that were upregulated in metastatic PCa MLL have a binding site in the 3'-UTR of CP110, which suggests that simultaneously with miR-129-3p other miRNAs may also contribute to the control of CP110 levels and centrosome function. miR-34 was previously confirmed to repress CP110 [19] and is also strongly upregulated in metastatic $\mathrm{PCa}$. We do not exclude a role for miR-34 or other miRNAs in the repression of CP110 in metastatic PCa (Supplementary Table S2), however functional experiments with miR-129-3p inhibitors clearly demonstrate a significant contribution of miR-129-3p to the expression of CP110.
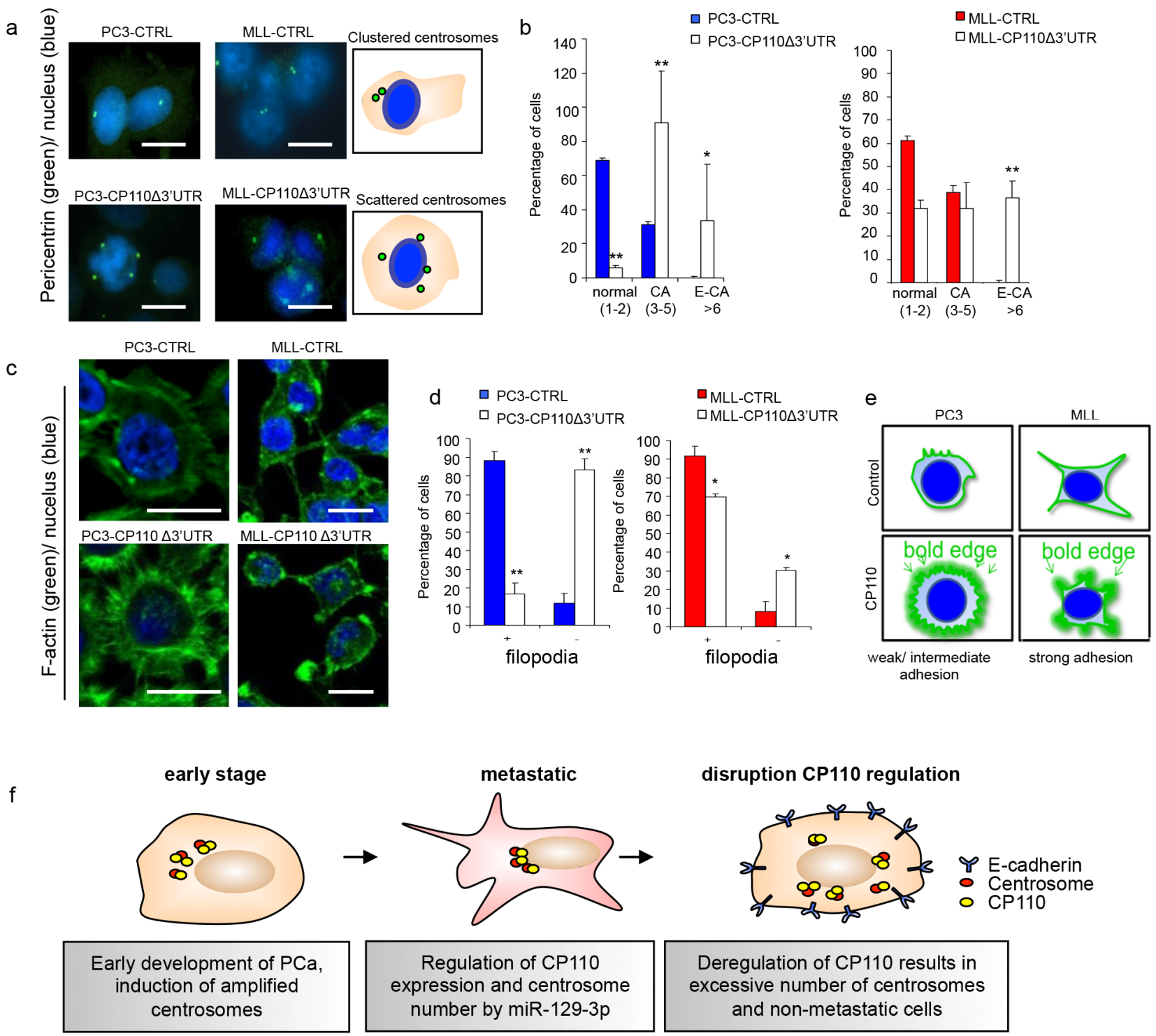

Regulation of CP110 expression and centrosome number by miR-129-3p disruption CP110 regulation

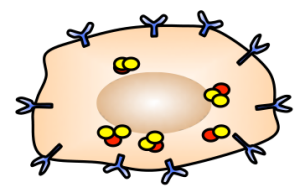

E-cadherin - Centrosome - CP110

Figure 5: CP110 regulates PCa invasion via centrosomes. a. Left panel cells stained for pericentrin (green) and DNA (hoechst, blue). Right panel schematic representation of pericentrin stained cells. b. Quantification of a). c. Cells stained for F-actin (green) and DNA (Hoechst, blue). d. Quantification of c). e. Schematic representation of F-actin stained cells. f. Schematic overview for the functions of miR-129-3p and CP110 in PCa metastasis. At an early stage, PCa cells show amplified centrosome number leading to a more aggressive phenotype. When cells enter a metastatic state, the centrosome number is regulated by miR-129-3p mediated repression of CP110. Upon overexpression of CP110, cells will increase centrosome number, E-cadherin expression leading to a non-metastatic phenotype. ${ }^{*} \mathrm{p}<0.05$, $* * \mathrm{p}<0.01$; T-test. 
We confirmed that PCa metastasis is accompanied by down-regulation of E-cadherin [31], resulting in cytoskeleton rearrangements and the formation of filopodia, typical for EMT [31]. Down-regulation of E-cadherin is an important hallmark of EMT, a process involved in the first steps of the tumor metastasis cascade [31, 32]. Although we observed a direct correlation between PCa metastasis, CP110 expression, and E-cadherin expression, we found no significant correlation between CP110 expression and the expression of EMT-related genes in four independent clinical PCa datasets. Hence, we suggest that centrosomal $\mathrm{CP} 110$ controls the metastatic process in PCa cells in an EMT-independent manner, as was recently also postulated for the role of centrosomes in the formation of breast cancer metastases [5]. CA is described to occur at early stages of cancer progression and has been related to increased tumor aggressiveness $[4,33,34]$. However, at a later stage in the metastatic process the relation between amplified centrosomes and metastasis remains unclear. Reports in which a relation between CA and metastasis is studied are conflicting [35]. CP110 expression has previously been described to lead to amplified centrosomes, and increased cell invasion in vitro [10]. However, the nature of CP110 and $\mathrm{CA}$ regulation in metastatic cancer has not been described. We demonstrate that CA (3-5 centrosomes/ cell) in PC3 and MLL cells is regulated through control of CP110 expression by miR-129-3p. CP110 overexpression and abrogation of its regulation by deletion of miR-129-3p binding site in CP110-3'UTR, resulted in an excess amplification of centrosomes, and reduced aggressiveness of prostate tumor cells. Most important for cancer cells to maintain their polarized potential is to correctly position the centrosomes within the cell [36]. This positioning is regulated by multiple proteins [36]. Moreover, an excess number of centrosomes can lead to disruption of cell migration by centrosome scattering $[37,38]$. In agreement with this, we observed a lack of centrosome clustering in CP110 overexpressing PCa cells in vitro and reduced metastatic potential in vivo. Importantly, the centrosome is emerging as a potential therapeutic target in cancer, including PCa [39]. Potential centrosomal drug targets include cyclin-dependent kinases, polo-like kinases [4042], aurora kinases [43] and molecular motor proteins [44]. Several small molecule inhibitors affecting centrosome function have been reported, of which Plk1 inhibitors are already tested in initial clinical studies [40, 45, 46]. While CP110 may also regulate ciliation, a process usually downregulated in (prostate) cancer cells [47], it remains to be investigated to what extent dysfunctional cilia contribute to PCa metastasis and the undirected migration of PCa cells.

In conclusion, we demonstrate that the centrosomal protein CP110 is at least partly regulated by miR-129-3p, and plays a functional role in PCa invasion and metastasis. Our results suggest that in metastatic PCa cells miR-129$3 \mathrm{p}$ is upregulated to reduce CP110 levels, preventing cells to develop an excess number of centrosomes. These results provide a functional link between aberrant centrosome function and PCa metastasis. Further research aimed at therapeutic targeting of the centrosome in metastatic PCa cells is warranted.

\section{MATERIALS AND METHODS}

\section{Cells}

Three stages of the Dunning model were selected for miRNA profiling studies, i.e. anaplastic tumor R3327AT (AT1; invasive growth, non-metastatic) and R3327MATLyLu tumors (MLL; metastatic) (Figure 1A). The tumor cells were expanded in male Copenhagen rats in vivo [48]. For in vitro studies, MLL and the human prostate cancer cell lines PC3 and LNCaP (ATCC) were cultured in RPMI 1640 culture medium, supplemented with $10 \%$ FCS and $50 \mathrm{U} / \mathrm{ml}$ penicillin and $50 \mu \mathrm{g} / \mathrm{ml}$ streptomycin. All cells were maintained in a $5 \% \mathrm{CO}_{2}$-humidified atmosphere at $37^{\circ} \mathrm{C}$. MLL-FM-CTRL cells were engineered by transduction with a lentivector encoding Firefly luciferase and mCherry as described elsewhere [49]. MLL-FM-CP110 43 'UTR cells were generated by stable transfection and selection of MLL-FM-CTRL cells with a CP110 rescue expression construct without miR129-3p 3'-UTR binding site (OriGene, Rockville, MD). PC3-CP110 $\Delta 3^{\prime}$ UTR cells were generated with the same CP110 rescue construct.

\section{miRNA profiling}

miRNA profiling of AT1 and MLL tumor tissue $(n=4)$ was performed by Exiqon (Vedbaek, Denmark). RNA was isolated from the tumor homogenates by Qiagen miRNA isolation kit (Qiagen). Total RNA quality was verified by an Agilent 2100 Bioanalyzer profile. 1 $\mu \mathrm{g}$ total RNA from sample and reference was labelled with Hy3 and Hy5 fluorescent label, respectively, using the miRCURY LNA Array power labelling kit (Exiqon, Denmark). The Hy3-labeled samples and a Hy5labeled reference RNA sample were mixed pair-wise and hybridized to the miRCURY LNA Array version $5^{\text {th }}$ Generation (Exiqon), which contains capture probes targeting 387 miRNAs for rat registered in miRBASE 15.0. The hybridization was performed using a Tecan HS4800 hybridization station (Tecan, Austria). The slides were scanned with the Agilent G2565BA Microarray Scanner System (Agilent Technologies, Inc., USA) and image analysis was carried out using the ImaGene 9.0 software (BioDiscovery, Inc., USA). The quantified signals were background corrected (Normexp with offset value 10) and normalized using the global Lowess (Locally 
Weighted Scatterplot Smoothing) regression algorithm. miRNAs were selected for significant $(p<0.05)$ up- or downregulation. RNA sequencing of human miRNAs expressed in cell cultures was performed according to Illumina protocols, as previously described [50]. For data analysis, we applied Illumina's software packages (SCS2.9/RTA1.9 and Off-line Basecaller v1.9).

\section{Patient archival prostate tissue samples}

Prostate tissues from 41 patients with adenocarcinoma of the prostate were evaluated for miRNA expression levels. The diagnosis was based on histopathological examination. Radical prostatectomies were performed between 1994 and 2011 at the Department of Urology, VU University Medical Center, Amsterdam, The Netherlands. Selection criteria were tumor present in the tissue blocks and prognosis. Postoperatively, the patients were scheduled for regular follow-up visit at the institutional outpatient clinic. Biochemical progression was defined as rising postoperative PSA levels by $>0.2$ $\mathrm{ng} / \mathrm{ml}$, confirmed in a consecutive visit. Biochemical recurrence was observed in 25 of the total of 41 patients.

\section{Transfection with miRNA mimics and inhibitors}

Cells were transfected by reverse transfection of miRNA precursors (mimics) or inhibitors or their controls (20 nM in optiMeM, Ambion, Austin), according to the manufacturer's instructions. For each experiment, the media were removed $24 \mathrm{~h}$ after transfection and replaced by regular culture media. The cells were cultured for another $24 \mathrm{~h}$ prior to use in experiments.

\section{Proliferation assay}

Cells were seeded (2,000 cells/well) for reverse transfection in 96-well plates (Greiner BioOne, Frickenhausen Germany). At $24 \mathrm{~h}$ after transfection, the medium was replaced by fresh culture medium. After 72 $\mathrm{h}$ the cells were fixed by trichloroacetic acid at $4^{\circ} \mathrm{C}$ for 1 $h$ and stained by sulphorhodamine B (SRB). The SRBdye was diluted in $10 \mathrm{mM}$ TRIS solution and the optical density (OD) was measured at 492-540 $\mathrm{nm}$ after which the OD of the controls was set to $100 \%$.

\section{Migration assay}

At $24 \mathrm{~h}$ after reverse transfection, the medium was replaced by fresh culture medium and $24 \mathrm{~h}$ later, the wound healing (scratch) assay was performed after uniformly scratching cellular monolayers using a guided 96-well pin tool (Peira, Turnhout, Belgium). Wells were washed, and fresh medium was added. Images were automatically captured at $\mathrm{t}=0$ and $\mathrm{t}=6 \mathrm{~h}$ on a Leica DMI3000 microscope (Leica, Rijswijk, The Netherlands) using Universal Grab 6.3 software (DCILabs, Keerbergen, Belgium). Scratch sizes were determined using Scratch Assay 6.2 (DCILabs). Wound closure $\left(\mu \mathrm{m}^{2}\right)$ was expressed as a percentage of that in control wells.

\section{Invasion assay}

The invasion assay was carried out using transwell chambers with a fluorescence-blocking $8 \mu \mathrm{m}$ pore filter insert (Becton Dickinson Labware, Bedford, MA, USA) as described previously [51]. In brief, the insert was coated with $5 \mathrm{ng}$ matrigel (Sigma-Aldrich Chemicals, Zwijndrecht, The Netherlands). Cells (200,000/insert) were seeded $48 \mathrm{~h}$ after transfection in serum free medium. In order to count the cells that have invaded after $24 \mathrm{~h}$, calcein-AM fluorescently labelled cells were counted.

\section{RT-PCR}

RNA from cell lines and frozen tumor tissues was extracted using a microRNeasy kit (Qiagen, Westburg, Leusden, The Netherlands) according to manufacturer's instructions. RNA for paraffin embedded tissues was extracted using Qiagen FFPE RNA isolation kit according to manufacturer's instructions. Subsequently, extracts were reverse transcribed using MultiScribe Reverse Transcriptase and the specific miRNA primer (Life Technologies) according to manufacturer's instructions. cDNA samples were amplified using a LightCycler 480 (Roche Diagnostics, Almere, The Netherlands) with 15 sec $95^{\circ} \mathrm{C}$ denaturation and $60 \sec 60^{\circ} \mathrm{C}$ primer annealing/ extension for 50 cycles starting with a 10 min hot start at $95^{\circ} \mathrm{C}$. In relative PCR quantifications, RNU-6B was used as endogenous control, and results are expressed in arbitrary units fold change compared to AT-1-tumor or LNCaP cells.

\section{Western blotting}

Whole cell protein was isolated by scraping in lysis buffer (Cell Signaling Technology, Inc, Danvers, MA, USA). Western blot was performed as described previously [51]. Briefly, from each condition $30 \mu \mathrm{g}$ of protein was separated on a 10\% SDS-PAGE and electroblotted onto polyvinylidenedifluoride (PVDF) membranes (Millipore Immobilon-FL PVDF, $0.45 \mu \mathrm{m}$ ). Subsequently, the membranes were blocked and incubated overnight at $4^{\circ} \mathrm{C}$ with the anti-CP110 (Cell Signaling, $1: 1,000)$, E-cadherin $(1: 500$; Novacastra, UK) or anti- $\beta$ actin-antibody (Sigma, dilution 1:10,000). The bands were scanned using an Odyssey Infrared Imager (Westburg), 84 $\mu \mathrm{m}$ resolution, $0 \mathrm{~mm}$ offset and with high quality.

\section{Immunofluorescent staining}

In 8-chamber labtek microscope slides, 100,000 cells were seeded. After $48 \mathrm{~h}$ cells were fixed in icecold methanol and blocked 30 min with $3 \%$ BSA (room 
temperature (RT)) and stained for either F-actin (Sigma) or pericentrin (provided by C. King, VU University, Amsterdam, NL) overnight at $4^{\circ} \mathrm{C}$. After washing, the secondary antibody was incubated (1:200, anti-mouseFITC, Dako) for $1 \mathrm{~h}$ at RT. The nucleus was stained with Hoechst 33342 (1:2,000) for $10 \mathrm{~min}$ at RT. Subsequently, slides were washed, and mounted onto microscope slides using vectashield (Vector, Burlingame, CA, USA). Stained cells were imaged on a Zeiss confocal LSM510 microscope (Carl Zeiss, Jena, Germany) using a x40 oil objective $(\mathrm{NA}=1.20)$. Imaged were analysed by Fiji software (Fiji, www.fiji.sc).

\section{In vivo metastasis}

Animal experiments were approved by the local committee on Animal Experiments of the VU University Medical Center, Amsterdam, the Netherlands. Male Copenhagen rats (age 2 months) were injected subcutaneously at the flank of the lower back with 500,000 cells/200 $\mu$ l of either MLL-FM-CTRL ( $n=7$ rats) or MLLFM-CP110 $\Delta 3^{\prime}$ UTR ( $=8$ rats) cells. Primary tumor growth was measured using a calliper and the volume was calculated using the formula: (length $\mathrm{x}$ width $\left.^{2}\right) / 2$. In addition presence of lymph node metastasis was measured weekly by measuring the Fluc signal by a charge-coupled device (CCD) camera, using the Xenogen-IVIS Lumina System (Xenogen Corp., Alameda, CA). Rats were injected intraperitoneally with $300 \mu \mathrm{l}$ D-Luciferin (200 $\mathrm{mg} / \mathrm{kg}$ body weight) under anaesthesia $\left(1.5 \mathrm{~L} \mathrm{O}_{2} /\right.$ minute and 3\% isoflurane). Regions of interest were defined in the abdomen where lymph node metastases were expected of which the local bioluminescence signals and background signals were subtracted. The photon counts $\left(\mathrm{p} / \mathrm{s} / \mathrm{cm}^{2}\right)$ in these regions were used as a total measurement of Fluc activity.

\section{Immunohistochemical staining of CP110}

Immunohistochemistry was performed on $3 \mu \mathrm{m}$ thick paraffin embedded sections of radical prostatectomy specimens. Slides were deparaffinised by steps of xylene and subsequently rehydrated by decreasing concentrations of ethanol. Endogenous peroxide expression was blocked by incubation with $0.3 \% \mathrm{H}_{2} \mathrm{O}_{2}$ in methanol for $30 \mathrm{~min}$. Slides were heated for antigen retrieval in $1 \mathrm{mM}$ Citrate buffer ( $\mathrm{pH}$ 6.0). Slides were blocked with $3 \% \mathrm{BSA}$ for $10 \mathrm{~min}$ at RT and exposed to the primary antibody against anti-CP110 (1:200, $\mathrm{O} / \mathrm{N}$ at $\left.4^{\circ} \mathrm{C}\right)$. After incubation, sections were washed and incubated with post-antibody blocking solution (Immunologic, Duiven, The Netherlands) for $15 \mathrm{~min}$ at RT. Subsequently, slides were washed and incubated with Brightvision-plus (Immunologic) for $60 \mathrm{~min}$ at RT. Peroxide was revealed by AEC solution (Invitrogen). Slides were counterstained with Hematoxylin and were covered in Kaisers glycerol (Merck KGAa, Darmstadt,
Germany). Blinded for the clinical parameters, including Gleason, progression and metastasis development, immunohistochemically stained slides were reviewed by both an experienced pathologist and senior researcher. CP110 staining of the tumor tissue section was scored for staining intensity (0-3).

\section{EMT analysis}

For analysis of the relation between CP110 and EMT genes, a previously reported EMT marker gene set was selected [27]. From this a weighed and directional gene signature was created using the appropriate prostate tumor, MAS5.0 normalized, mRNA expression sets using the R2 program (http://r2.amc.nl). Expression of CP110 expression was correlated with this weighed and averaged signature score. Datasets that were selected for analysis include: ExpO (http://www.intgen.org), Jenkins-545 [23], Sueltman [24], Ethnic-89-MAS5.0-u133a [25] and Svensson-108-MAS5.0-u133a [26].

\section{Statistical analysis}

For calculating significant differences between the parental and the transfected cells or between treated and untreated samples, the two-tailed Student's t-test was used. The values were considered significantly different when $\mathrm{p}<0.05$. Chi-square test was used for calculation of significant differences between patient groups of metastasis and non-metastasis and/or high and low Gleason score, using SPSS 20.0. Groups were found to be statistically different when $\mathrm{p}<0.05$.

\section{ACKNOWLEDGMENTS}

Stichting Pon, Stichting VUmc CCA and the Worldwide Cancer Research Foundation (AICR \#151005 ) are kindly acknowledged for financially supporting the present study.

\section{CONFLICTS OF INTEREST}

The authors declare no conflict of interest.

\section{REFERENCES}

1. Siegel R, Ma J, Zou Z, Jemal A. Cancer statistics, 2014. CA. Cancer J. Clin. 2014; 64: 9-29.

2. Fidler IJ. The pathogenesis of cancer metastasis: the "seed and soil" hypothesis revisited. Nat. Rev. Cancer. 2003; 3: 453-458.

3. Bettencourt-Dias M, Glover DM. Centrosome biogenesis and function: centrosomics brings new understanding. Nat. Rev. Mol. Cell Biol. 2007; 8: 451-463.

4. Zyss D, Gergely F. Centrosome function in cancer: guilty or innocent? Trends Cell Biol. 2009; 19: 334-346. 
5. Godinho SA, Picone R, Burute M, Dagher R, Su Y, Leung CT, Polyak K, Brugge JS, Théry M, Pellman D. Oncogenelike induction of cellular invasion from centrosome amplification. Nature. 2014; 510: 167-71.

6. Sluder G, Nordberg JJ. The good, the bad and the ugly: The practical consequences of centrosome amplification. Curr. Opin. Cell Biol. 2004; 16: 49-54.

7. D'Angiolella V, Donato V, Vijayakumar S, Saraf A, Florens L, Washburn MP, Dynlacht B, Pagano M. $\mathrm{SCF}(\mathrm{Cyclin} \mathrm{F})$ controls centrosome homeostasis and mitotic fidelity through CP110 degradation. Nature. 2010; 466: 138-142.

8. Franz A, Roque H, Saurya S, Dobbelaere J, Raff JW. CP110 exhibits novel regulatory activities during centriole assembly in Drosophila. J. Cell Biol. 2013; 203: 785-799.

9. Tsang WY, Spektor A, Luciano DJ, Indjeian VB, Chen Z, Salisbury JL, Sánchez I, Dynlacht BD. CP110 cooperates with two calcium-binding proteins to regulate cytokinesis and genome stability. Mol. Biol. Cell. 2006; 17: 3423-3434.

10. Cao J, Shen Y, Zhu L, Xu Y, Zhou Y, Wu Z, Li Y, Yan X, Zhu X. miR-129-3p controls cilia assembly by regulating CP110 and actin dynamics. Nat. Cell Biol. 2012; 14: 697-706.

11. Li J, D’Angiolella V, Seeley ES, Kim S, Kobayashi T, Fu W, Campos EI, Pagano M, Dynlacht BD. USP33 regulates centrosome biogenesis via deubiquitination of the centriolar protein CP110. Nature. 2013; 495: 255-9.

12. Flynt AS, Lai EC. Biological principles of microRNAmediated regulation: shared themes amid diversity. Nat. Rev. Genet. 2008; 9: 831-842.

13. Lujambio A, Lowe SW. The microcosmos of cancer. Nature. 2012; 482: 347-355.

14. Wang G, Wang Y, Feng W, Wang X, Yang JY, Zhao Y, Wang Y LY. Transcription factor and microRNA regulation in androgen-dependent and -independent prostate cancer cells. BMC Genomics. 2008; 9.

15. Varambally S, Cao Q, Mani R-S, Shankar S, Wang X, Ateeq B, Laxman B, Cao X, Jing X, Ramnarayanan K, Brenner JC, Yu J, Kim JH et al. Genomic loss of microRNA-101 leads to overexpression of histone methyltransferase EZH2 in cancer. Science. 2008; 322: 1695-1699.

16. Schaefer A, Jung M, Mollenkopf H-J, Wagner I, Stephan C, Jentzmik F, Miller K, Lein M, Kristiansen G, Jung K. Diagnostic and prognostic implications of microRNA profiling in prostate carcinoma. Int. J. Cancer. 2010; 126: 1166-1176.

17. Martens-Uzunova ES, Jalava SE, Dits NF, van Leenders GJLH, Møller S, Trapman J, Bangma CH, Litman T, Visakorpi T, Jenster G. Diagnostic and prognostic signatures from the small non-coding RNA transcriptome in prostate cancer. Oncogene. 2012; 31: 978-991.

18. Wang L, Tang H, Thayanithy V, Subramanian S, Oberg AL, Cunningham JM, Cerhan JR, Steer CJ, Thibodeau SN. Gene networks and microRNAs implicated in aggressive prostate cancer. Cancer Res. 2009; 69: 9490-9497.
19. Song R, Walentek P, Sponer N, Klimke A, Lee JS, Dixon G, Harland R, Wan Y, Lishko P, Lize M, Kessel M, He L. miR-34/449 miRNAs are required for motile ciliogenesis by repressing cp110. Nature. 2014; 510: 115-120.

20. Tennant TR, Kim H, Sokoloff M, Rinker-Schaeffer CW. The Dunning model. Prostate. 2000; 43: 295-302.

21. Dedhar S, Saulnier R, Nagle R OC. Specific alterations in the expression of alpha 3 beta 1 and alpha 6 beta 4 integrins in highly invasive and metastatic variants of human prostate carcinoma cells selected by in vitro invasion through reconstituted basement membrane. Clin. Exp. Metastasis. 1993; 11: 391-400.

22. Wu X, Gong S, Roy-Burman P, Lee P, Culig Z. Current mouse and cell models in prostate cancer research. Endocr Relat Cancer. 2013; 20: R155-70.

23. Erho N, Crisan A, Vergara IA, Mitra AP, Ghadessi M, Buerki C, Bergstralh EJ, Kollmeyer T, Fink S, Haddad Z, Zimmermann B, Sierocinski T, Ballman K V. et al. Discovery and Validation of a Prostate Cancer Genomic Classifier that Predicts Early Metastasis Following Radical Prostatectomy. PLoS One. 2013; 8.

24. Börno ST, Fischer A, Kerick M, Fälth M, Laible M, Brase JC, Kuner R, Dahl A, Grimm C, Sayanjali B, Isau M, Röhr $\mathrm{C}$, Wunderlich A et al. Genome-wide DNA methylation events in TMPRSS2-ERG fusion-negative prostate cancers implicate an EZH2-dependent mechanism with miR-26a hypermethylation. Cancer Discov. 2012; 2: 1024-1035.

25. Wallace TA, Prueitt RL, Yi M, Howe TM, Gillespie JW, Yfantis HG, Stephens RM, Caporaso NE, Loffredo CA, Ambs S. Tumor immunobiological differences in prostate cancer between African-American and European-American men. Cancer Res. 2008; 68: 927-936.

26. Svensson JP, Stalpers LJ, Esveldt-van Lange RE, Franken NA, Haveman J, Klein B, Turesson I, Vrieling H, GiphartGassler M. Analysis of gene expression using gene sets discriminates cancer patients with and without late radiation toxicity. PLoS Med. 2006; 3: e422.

27. Li P, Yang R, Gao W-Q. Contributions of epithelialmesenchymal transition and cancer stem cells to the development of castration resistance of prostate cancer. Mol. Cancer. 2014; 13: 55.

28. Shimizu H, Nagamori I, Yabuta N, Nojima H. GAK, a regulator of clathrin-mediated membrane traffic, also controls centrosome integrity and chromosome congression. J. Cell Sci. 2009; 122: 3145-3152.

29. Marthiens V, Piel M, Basto R. Never tear us apart - the importance of centrosome clustering. J. Cell Sci. 2012; 125: 3281-3292.

30. Rida PCG, Cantuaria G, Reid MD, Kucuk O, Aneja R. How to be good at being bad: centrosome amplification and mitotic propensity drive intratumoral heterogeneity. Cancer Metastasis Rev. 2015 in press.

31. Lamouille S, Xu J, Derynck R. Molecular mechanisms of epithelial-mesenchymal transition. Nat. Rev. Mol. Cell Biol. 2014; 15: 178-96. 
32. De Craene B, Berx G. Regulatory networks defining EMT during cancer initiation and progression. Nat. Rev. Cancer. 2013; 13: 97-110.

33. Ouyang X, Wang X, Xu K, Jin DY, Cheung ALM, Tsao SW, Wong YC. Effect of p53 on centrosome amplification in prostate cancer cells. Biochim. Biophys. Acta - Mol. Cell Res. 2001; 1541: 212-220.

34. Li J, Xuan JW, Khatamianfar V, Valiyeva F, Moussa M, Sadek A, Yang BB, Dong BJ, Huang YR, Gao WQ. SKA1 over-expression promotes centriole over-duplication, centrosome amplification and prostate tumourigenesis. Journal of Pathology. 2014; 234: 178-89

35. Chan JY. A clinical overview of centrosome amplification in human cancers. Int. J. Biol. Sci. 2011; 7: 1122-1144.

36. Tonucci FM, Hidalgo F, Ferretti a., Almada E, Favre C, Goldenring JR, Kaverina I, Kierbel a., Larocca MC. Centrosomal AKAP350 and CIP4 act in concert to define the polarized localization of the centrosome and Golgi in migratory cells. J. Cell Sci. 2015; 128: 3277-3289.

37. Kushner EJ, Ferro LS, Liu JY, Durrant JR, Rogers SL, Dudley AC, Bautch VL. Excess centrosomes disrupt endothelial cell migration via centrosome scattering. J. Cell Biol. 2014; 206: 257-272.

38. Ganem NJ, Godinho SA, Pellman D. A mechanism linking extra centrosomes to chromosomal instability. Nature. 2009; 460: 278-282.

39. Yanag, T, Krajewska M, Matsuzawa SI RJ. PCTAIRE1 phosphorylates p27 and regulates mitosis in cancer cells. Cancer Res. 2014; 74: 5795-807.

40. Christoph DC, Schuler M. Polo-like kinase 1 inhibitors in mono- and combination therapies: a new strategy for treating malignancies. Expert Rev. Anticancer Ther. 2011; 11: $1115-1130$.

41. Bruinsma W, Raaijmakers JA, Medema RH. Switching Polo-like kinase-1 on and off in time and space. Trends Biochem. Sci. 2012; 37: 534-542.

42. Korzeniewski N, Hohenfellner M, Duensing S. The centrosome as potential target for cancer therapy and prevention. Expert Opin. Ther. Targets. 2013; 17: 43-52.
43. Malumbres M P de CI. Aurora kinase A inhibitors: promising agents in antitumoral therapy. Expert Opin Ther Targets. 2014; 18: 1377-93.

44. Nam H-J, van Deursen JM. Cyclin B2 and p53 control proper timing of centrosome separation. Nat. Cell Biol. 2014; 16: 538-49.

45. Lewis SR, Hedman CJ, Ziegler T, Ricke WA, Jorgensen JS. Steroidogenic factor 1 promotes aggressive growth of castration-resistant prostate cancer cells by stimulating steroid synthesis and cell proliferation. Endocrinology. 2014; 155: 358-369.

46. Hou X, Li Z, Huang W, Li J, Staiger C, Kuang S, Ratliff T, Liu X. Plk1-dependent microtubule dynamics promotes androgen receptor signaling in prostate cancer. Prostate. 2013; 73: 1352-1363.

47. Hassounah NB, Nagle R, Saboda K, Roe DJ, Dalkin BL, McDermott KM. Primary Cilia Are Lost in Preinvasive and Invasive Prostate Cancer. PLoS One. 2013; 8: e68521.

48. Geldof AA, Van Den Tillaar PLM, Newling DWW, Teule GJJ. Radionuclide therapy for prostate cancer lumbar metastasis prolongs symptom-free survival in a rat model. Urology. 1997; 49: 795-801.

49. Wurdinger T, Badr C, Pike L, de Kleine R, Weissleder R, Breakefield XO, Tannous BA. A secreted luciferase for ex vivo monitoring of in vivo processes. Nat. Methods. 2008; 5: 171-173.

50. Koppers-Lalic D, Hackenberg M, Bijnsdorp I, van Eijndhoven M, Sadek P, Ylstra B, Middeldorp J, Sie D, de Menezes R, Würdinger T, Meijer G, Pegtel D. Nontemplated Nucleotide Additions distinguish the Small RNA composition in Cells from Exosomes. Cell Rep. 2014; 8: 1649-58.

51. Bijnsdorp I V, Rozendaal L, van Moorselaar RJ, Geldof AA. A predictive role for noncancerous prostate cells: low connexin-26 expression in radical prostatectomy tissues predicts metastasis. Br. J. Cancer. 2012; 107: 1963-8. 